\title{
Sleeved Bending Actuators for Soft Grippers: A Durable Solution for High Force-to-Weight Applications
}

\author{
Geneviève Miron *, Benjamin Bédard and Jean-Sébastien Plante * \\ Département de génie mécanique, Université de Sherbrooke, Sherbrooke, QC J1K 2R1, Canada; \\ benjamin.bedard@usherbrooke.ca \\ * Correspondence: genevieve.miron@usherbrooke.ca (G.M.); jean-sebastien.plante@usherbrooke.ca (J.-S.P.); \\ Tel.: +819-821-8000 (G.M. \& J.-S.P.)
}

Received: 6 June 2018; Accepted: 13 July 2018; Published: 17 July 2018

\begin{abstract}
Soft grippers are known for their ability to interact with objects that are fragile, soft or of an unknown shape, as well as humans in collaborative robotics applications. However, state-of-the-art soft grippers lack either payload capacity or durability, which limits their use in industrial applications. In fact, high force density pneumatic soft grippers require high strain and operating pressure, both of which impair their durability. This work presents a new sleeved bending actuator for soft grippers that is capable of high force density and durability. The proposed actuator is based on design principles previously proven to improve the life of pneumatic artificial muscles, where a sleeve provides a uniform reinforcement that reduces local stresses and strains in the inflated membrane. The sleeved bending actuator features a silicone membrane and an external two-material sleeve that can support high pressures while providing a flexible grip. The proposed sleeved bending actuators are validated through two grippers, sized according to foreseen soft gripper applications: A small gripper for drone perching and lightweight food manipulation, and a larger one for the manipulation of heavy material $(>5 \mathrm{~kg}$ ) of various weights and sizes. Performance assessment shows that these grippers have payloads up to $5.2 \mathrm{~kg}$ and $20 \mathrm{~kg}$, respectively. Durability testing of the grippers demonstrates that the grippers have an expected lifetime ranging from 263,000 cycles to more than 700,000 cycles. The grippers are tested in various settings, including the integration of a gripper into a Phantom 2 quadcopter, a perching demonstration, as well as the gripping of light and heavy food items. Experiments show that sleeved bending actuators constitute a promising avenue for durable and strong soft grippers.
\end{abstract}

Keywords: Soft robotics; gripper; bending actuator; fatigue; elastomers

\section{Introduction}

\subsection{Motivation}

Soft grippers' unique characteristics of compliance, resistance to impact, and lightness make them more relevant than ever for applications requiring robot-human interaction or grasping objects of uneven shapes or in unknown environments. Existing soft grippers rely on various technologies, including granular jamming [1], underactuated cable fingers [2], bending fluidic muscles [3], electroadhesive sheets [4], etc. Among soft gripper technologies, the most interesting with regard to force density are shape-memory alloys (payload-to-weight ratio up to 925 [5]), electroadhesion (ratio up to 54 [4]), geckoadhesion (ratio up to 286 [6]), and fluidic actuators (up to ratios of 68 [7]). These impressive densities are, however, counterbalanced by a low frequency and high hysteresis (shape memory alloys) or high dependence on object surface (adhesion technologies). Fluidic grippers show a great balance of simplicity, low cost, strength, and low weight, which makes them a popular 
choice among soft robotics grippers [8]. Fluidic grippers have been showcased in various applications, such as industrial pick-and-place [9], manipulation of delicate biological samples from reefs [10], hand rehabilitation [3], etc.

However, widespread implementation of soft fluidic actuators is limited by the difficulty to model and control non-linear elastomer materials, as well as the compromise between force output and durability. This compromise translates into most soft fluidic grippers being designed for small loads (under $1 \mathrm{~kg}$ ), made of ultra-soft materials, and operated at low operating pressures. Most studies focus on developing new applications and efficient designs, while durability studies of soft fluidic grippers are almost non-existent. This paper aims to provide a possible solution to the force/durability compromise of soft pneumatic grippers through a new sleeved bending actuator, enabling payloads of up to $20 \mathrm{~kg}$ and withstanding over 700,000 cycles. The sleeve design is based on design principles previously shown to increase the life of high-strain pneumatic muscles [11].

\subsection{Background}

Soft grippers use actuators that are designed to adapt to objects of various shapes, textures, and rigidity by their constitution (soft material) and actuation means (cable, fluid, etc.). Fluidic actuators, especially, are designed so that the pressurization of one or more chambers(s) generates a bending (or twisting) motion. The actuator can be made of an elastomer or of bellows that deform under pressure. The pressure is transformed into usable work through the deformation of the actuator and, when the actuator is constrained by an object, into a force acting on the object being grasped. Bending actuators usually feature asymmetric reinforcements: a strain-limiting layer on the inside and a form of radial constraint on the outside (Figure 1). Bending actuators are divided into two categories with respect to their radial reinforcement: Monolithic polymer construction (geometrical features, Figure 1a) and fiber-reinforced construction (Figure 1b).

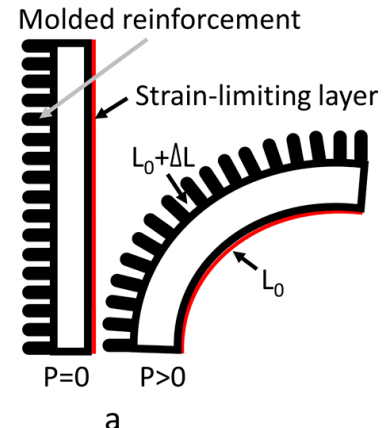

a

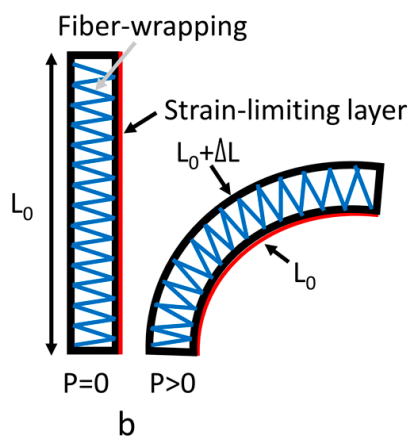

Figure 1. Working principle of soft bending actuators. (a) Monolithic polymer construction; (b) Fiberreinforced bending actuator.

\subsubsection{Monolithic Polymer Construction}

Geometrically constrained bending actuators consist of a molded elastomer part with a geometry that varies along the length of the actuator (Figure 1a), or with an asymmetric chamber with regard to the exterior of the actuator.

The gripper, developed by the company Soft Robotics [9], is intended as an industrial end-effector for pick-and-place applications, especially for the food industry, where the various shapes and fragility of the food benefit from the soft robotics approach $[12,13]$. The actuators are molded in a concertinaed manner to facilitate bending and minimize stretches. The various declinations of this gripper can grasp objects up to $4.5 \mathrm{~kg}$, demonstrate reverse grasping, and reach frequencies up to 120 picks per minute (data taken from the application videos available online [9]). Durability data are not published.

In another embodiment, ribbed 3D-printed bending fingers are assembled into a gripper that can hold a maximum payload of $5 \mathrm{~kg}$ [14], with a payload-to-weight ratio of 18 . The fatigue life of this 
concept is limited to 600 cycles at $250 \mathrm{kPa}$ (operating pressure). The recent development of highly stretchable and printable elastomers makes for an interesting future for 3D-printed bending fingers if their durability can be further increased to over a few thousands cycles [15]. Another pneumatic gripper [16] integrates electroadhesion into bending fingers, allowing the gripping of soft and flat objects as well large and regular objects. This gripper is well suited to the manipulation of light $(<100 \mathrm{~g})$ and delicate objects, although it is not conceived to hold large loads.

PneuNets (pneumatic networks), originally developed at Harvard, are bellowed actuators made by creating a network of pneumatic chambers inside a polymer structure. PneuNets bending actuators, intended for an assistive glove [3], are molded into a teethed shape on one side and reinforced with a strain-limiting fabric to provide a highly flexible structure. A variation of the PneuNets design is used as a gripper to collect delicate samples from reefs [10]. These actuators can reach large bending deformations in the order of $270^{\circ}$, but demonstrate payloads below $2 \mathrm{~kg}$ [10], and their durability has not been tested. Single-bodied grippers made of embedded pneumatic networks were also developed and tested to resist mechanical damage, such as being rolled on by a car [17]. These structures cannot withstand a large number of operation cycles because they undergo large deformations and high local stresses due to constraining structures.

Other PneuNets actuators were specifically designed for rapid actuation by minimizing the air volume inside and the deformation of the outside wall [18]. Thus, this reduced the operating pressure and material strain, increasing the life from under 1000 cycles to more than a million. However, the low operating pressure (72 $\mathrm{kPa})$ makes for a low pushing force $(1.4 \mathrm{~N}$ at the end of the actuator) and consequently a low payload.

All these actuators, restrained by their molded geometry, are fundamentally limited in pressure (and, thus, in payload) by the material's resistance and stretch. An approach to increase the pressure and, thus, the payload is to radially reinforce the polymer structure with an external reinforcement.

\subsubsection{Fiber Reinforcements}

Fiber-reinforced bending actuators are made by wrapping an elastomer tube (usually a half-cylinder) with fibers, such as a Kevlar threads to constrain the actuator radially (Figure 1b). The actuator has an overmolded elastomer layer to keep the fibers in place, creating an orthotropic composite material. The inside surface features a strain-limiting layer, creating a bending motion when pressurized [19]. A variation of this design integrates partial inextensible sleeves that affect the bending radius, allowing two $45 \mathrm{~g}$ fingers to support $6.1 \mathrm{~kg}$ [7]. The durability of these actuators has not been studied, but the fibers used as reinforcements cause highly localized strains and local abrasion, two phenomena that are known to cause premature membrane failure [11]. Other actuators are wrapped following a spiral pattern, and the actuator thus adopts a boa-like behavior (spiral deformation) under pressure. These actuators, developed for reef-sample collection, show payloads of up to $6 \mathrm{~kg} \mathrm{[10].} \mathrm{A} \mathrm{modular} \mathrm{bending} \mathrm{actuator} \mathrm{has}$ also been developed using polyurethane-coated fabric pockets assembled on a 3D-printed spine [20]. This was experimentally shown to sustain 100,000 cycles while producing a torque proportional to the pressure. An extensible pneumatic actuator using a McKibben-type braid was transformed into a bending actuator by adding a longitudinal inextensible fiber for a hand exoskeleton application [21]. The braid allows for operating pressures of up to $500 \mathrm{kPa}$, resulting in tip forces of up to $4.3 \mathrm{~kg}$.

In terms of durability, fatigue challenges of fiber-reinforced bending actuators are similar to those encountered by McKibben-type contractile muscles, since actuator durability is highly dependent on the fiber/membrane interaction mechanics [22,23]. McKibbens can undergo up to 120 million cycles for a maximal engineering strain of 5\% [24]. However, their durability drops between 20,000 and 100,000 cycles when deformations climb to 30\%, including commercial actuators [22-25]. Highly general strains, combined with local strains and abrasion caused by the interaction of the membrane with its reinforcement, lead to the formation of cracks that eventually cause failure. Fiber-reinforced bending actuators have a similar failure mechanism, since they also comprise a membrane that is pressurized against a fiber reinforcement, causing large local strains. 
In sum, there is still work to be done in designing fluidic actuators for soft grippers that are both strong and durable. Fatigue experiments have shown that the key to such strong and durable soft fluidic actuators is the design and integration of reinforcements that will minimize damage to the membrane [11].

\subsection{Approach}

In this paper, we present a soft robotic gripper with pneumatic bending fingers that can hold large payloads and resist a high number of actuation cycles. The fingers are designed using a sleeve concept based on principles to increase the life of fluidic extensible muscles [11]. The sleeve replaces the usual fiber-wrapping by a continuous external reinforcement, limiting local strains in the membrane, which is strong enough to support the pressures required for higher payloads. Requirements are taken from two promising applications of soft grippers: Drone perching and industrial manipulation. A first order model is used to predict the bending actuator workspace and estimate the payload capacity. A smaller, lighter gripper and a larger, stronger version are presented and validated through multiple applications, including the perching of a Phantom 2 quadcopter and food manipulation. The performance of both versions is validated through free bending, payload, and fatigue-life experiences. These experiments demonstrate that sleeved bending actuators make for strong, light, affordable, and reliable soft grippers. Compared to existing soft grippers, the proposed approach simultaneously allows for a large payload-to-weight ratio and a relevant fatigue life.

\section{Design}

\subsection{Bending Actuator Design}

Bending pneumatic actuators are designed following the general principle of having the inside surface restrained by a strain-limiting layer and the outside constrained radially, while allowing axial extension (Figure 1). Most existing bending muscles rely on a multistep molding process or fiber wrapping to create an orthotropic composite material $[3,9,19]$. Here, the design of bending actuators is based on previously published sleeved extensible pneumatic muscles, specifically designed to provide large strains (50\% engineering strain) while maintaining a fatigue life over 200,000 cycles [11]. The sleeve concept was developed with respect to the following three fatigue principles:

- $\quad$ Reducing local stresses and strains;

- Reducing surface damage (abrasion and cutting);

- Operating below the material's fatigue limit (strain under which the fatigue life tends towards infinity).

The bending actuator uses a commercially available silicone tube, made of a material tested to have a fatigue limit of over $50 \%$ (engineering strain), as the membrane [11]. A bending motion is produced by a two-material sleeve made from a woven elastic band (Figure 2a), commonly used in articles of clothing, which is sewn into an inelastic nylon-fabric strip (Figure $2 b, c)$. The nylon fabric acts as the strain-limiting layer, and the elastic band restrains radial inflation while allowing the outside of the membrane to stretch longitudinally. During assembly, the sleeve (Figure 2c) is slid over the membrane (Figure 2d) and clamped at both ends on end caps (Figure 2e,f). When the actuator is pressurized, the sleeve creates a bending deformation towards the strain-limiting layer. Maximum strain is reached on the outside of the bend, and minimum strain (near zero) at the seam of the strain-limiting layer. Compared to Kevlar fibers, commonly used in state-of-the-art fiber-reinforced bending actuators, the sleeve provides a large contact area and minimizes membrane indentation under pressure, thus limiting strain concentrations and surface damage. Furthermore, the sleeve resists much higher pressures than the polymer alone (previous tests showed that a constrained sleeved actuator endured a pressure of $650 \mathrm{kPa}$ without bursting). The sleeve also acts as a protecting layer over the membrane, avoiding puncture from sharp objects in the environment (e.g., rocks, marine life, thorny fruits, etc.). 


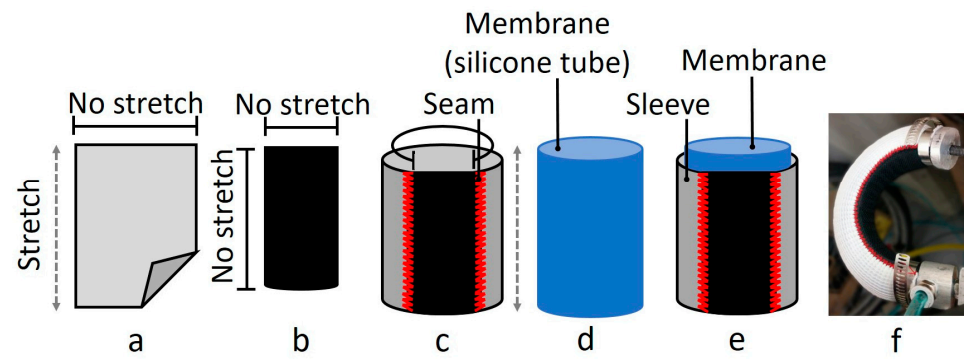

Figure 2. Assembly of a bending actuator. (a) Elastic band; (b) Strain-limiting layer; (c) Sleeve assembly; (d) Silicone tube, acting as the membrane; (e) Muscle assembly; and (f) Assembled bending muscle with end caps (pressurized).

\subsection{Requirements}

Requirements for both grippers are derived for two applications, consisting of drone perching and industrial manipulation.

- Drone perching (small gripper): A gripper allows a drone to perch on tree branches, fences, pipes, and power lines. Drone perching is useful for still surveillance operations or charging (solar electricity harvesting), both of which increase drone autonomy, a major challenge for long-haul missions [26]. The Phantom 2 quadcopter, chosen for this case study, has a $400 \mathrm{~g}$ payload (recommended for stable flight) that makes minimizing weight a critical requirement.

- Industrial manipulation (large gripper): A soft gripper for robotic manipulation can manipulate heavy common objects $(10 \mathrm{~kg})$ of all sorts of shapes and textures, such as potato bags, metal billets, etc. The maximum mass of the gripper is set to $600 \mathrm{~g}$ in order to reach a minimum payload-to-weight ratio of 15 .

\subsection{Grip}

The chosen grip scheme for the gripper is an enclosed power grip, meaning that the fingers close on themselves to grasp an object. This grip is mostly appropriate for cylindrical-like objects and provides the largest grip force [27]. The length of fingers is chosen to reproduce the gripping capacity of a human, which consists of cylindrical objects with a diameter of between $6 \mathrm{~cm}$ and $10 \mathrm{~cm}$ for heaviest loads [28]. This size is also appropriate for grasping most common objects: Tree branches, fences, mugs, automotive parts, etc. Another major factor in the design of efficient flexion fingers is the friction surface on the inside of the finger. Silicone strips are added to the nylon section of the sleeve to increase its friction coefficient with grasped objects.

\subsection{Detailed Geometry}

Finger geometry (Table 1) is chosen to provide large bending (using the model of Section 3) while considering available elastic-band widths, given the fact that elastic bands cannot be cut lengthwise without affecting their integrity.

The grippers are assembled by fixing the fingers on a "palm" (Figure 3). The small gripper, for weight considerations, has three fingers in a 2-1 facing configuration with a 3D-printed ABS (acrylonitrile butadiene styrene) palm. The larger gripper can accommodate three or four fingers in an offset facing configuration, but all experiments are performed with three fingers in a 2-1 facing configuration for comparison purposes. 
Table 1. Finger specifications for both the small and large versions.

\begin{tabular}{ccc}
\hline & Small & Large \\
\hline Membrane material & Silicone (McMaster-Carr \#5236K525) & Silicone (McMaster-Carr \#5236K234) \\
Outside diameter & $16 \mathrm{~mm}(5 / 8 \mathrm{in})$ & $22 \mathrm{~mm}(7 / 8 \mathrm{in})$ \\
Durometer & 35 Shore A & 50 Shore A \\
Elastic band width ${ }^{1}$ & $38 \mathrm{~mm}(1.5 \mathrm{in})$ & $57 \mathrm{~mm}(2.25 \mathrm{in})$ \\
Nylon band width & $15 \mathrm{~mm}$ & $20 \mathrm{~mm}$ \\
Length & $120 \mathrm{~mm}$ & $120 \mathrm{~mm}$ \\
Gripper mass (3 fingers) & $112.6 \mathrm{~g}$ & $594 \mathrm{~g}$ \\
\hline
\end{tabular}

${ }^{1}$ Woven elastic bands are used in common articles of clothing, and can be bought in bulk (unbranded) from fabric stores, or online on eBay (San Jose, CA, USA). A branded example is style W11L from the company Elastic Cord \& Webbing Inc. (East Dundee, IL, USA).

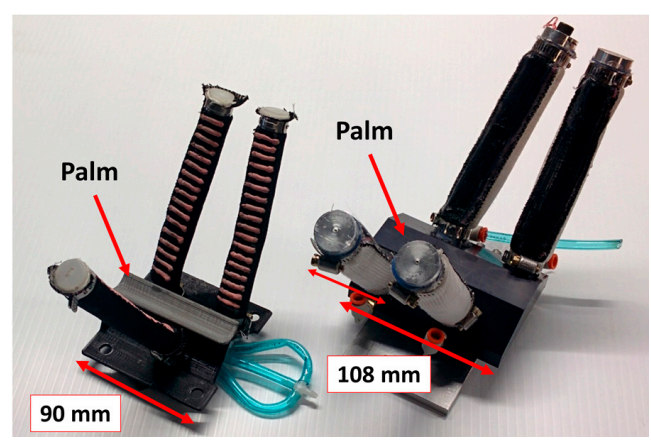

Figure 3. Assembled grippers. Left: Small; Right: Large.

\section{Actuator Model}

\subsection{Bending Model}

Actuator bending is modeled using a simple and comprehensive analytical model that allows quick scaling and comparison of the performance of new designs. The main objective of the model is to predict fingers' curvature radius and angle for various inputs, including pressure, composition (e.g., membrane material), and geometry (e.g., ratio of strain-limiting layer to elastic band, membrane diameter).

The bending finger is modeled as a beam with one fixed extremity and a free extremity subject to a bending moment $M_{\text {ext }}$ (Figure 4). Because the nylon fabric is inelastic ("infinite" stiffness), the inside surface of the finger has a constant length, and the neutral axis is supposed to be located at the seam. The external moment $M_{\text {ext }}$ is created by the pressure applied on the end face of the actuator. The external moment is compensated by an internal bending moment $M_{\text {int }}$, i.e., the finger's resistance to bending, and depends on material properties, section inertia, and bending radius.

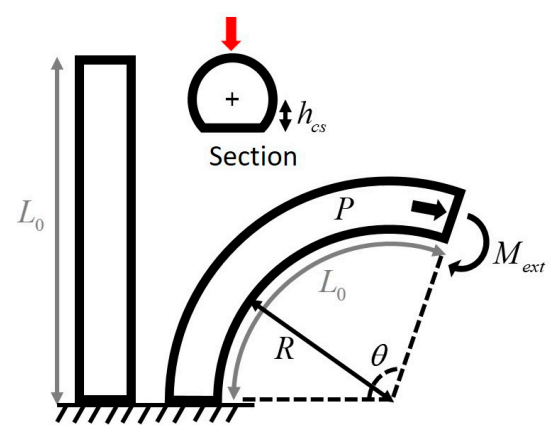

Figure 4. Main variables of the bending-actuator model. The red arrow indicates the location of maximum strain. 
Internal and external bending moments are defined by:

$$
\begin{gathered}
M_{\mathrm{int}}=\frac{E I}{R} \\
M_{\mathrm{ext}}=P A h_{c s}
\end{gathered}
$$

where $E$ is the approximate Young's modulus of the membrane based on the material's durometer [29], $I$ is the section's second moment of inertia, $R$ is the constant bending radius relative to the neutral axis (seam), $P$ the internal pressure, $A$ is the section area (internal), and $h_{\mathcal{~ S}}$ is the height of the section's area center from the neutral axis (pressure center). The section area is taken as $10 \%$ larger when the actuator is inflated due to sleeve and seam stretching (based on experiments, under all operating pressures, once the actuator has been stretched for more than 10 cycles at $310 \mathrm{kPa}$ ), and the sleeve's stiffness is considered negligible compared with the membrane's.

At equilibrium, the external and internal bending moments are equal. The bending radius can thus be found by combining Equations (1) and (2):

$$
R=\frac{E I}{P A h_{c s}}
$$

The length of the inside surface of the finger, $L_{0}$, is constant and allows the bending angle $\theta$ to be found through the equation for an arc's length:

$$
R \theta=L_{0}
$$

Using the model, $R$ and $\theta$ are calculated under pressures between 0 and $350 \mathrm{kPa}$ (Figure 5).

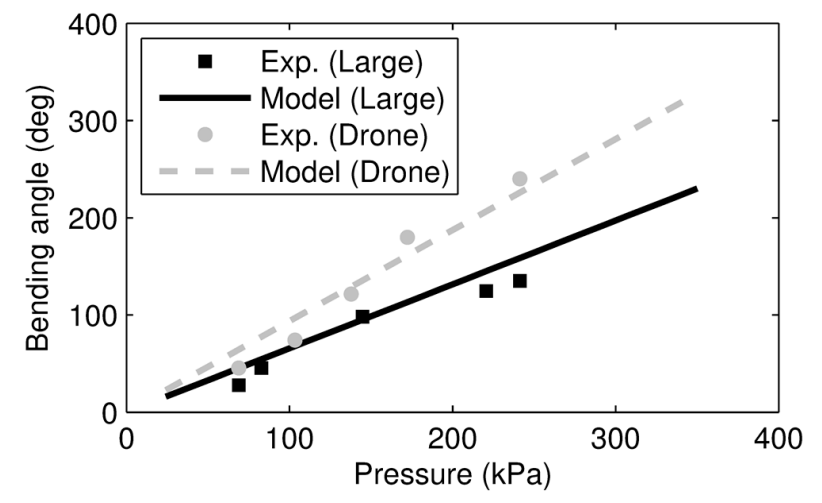

Figure 5. Modeled and experimental bending of bending actuators at various actuation pressures.

To validate the model, the actuator's bending radius and angle are measured by image analysis at various pressures (Figure 6). Bending is compared with model predictions to verify its accuracy by tracing the bending angle as a function of the applied pressure (Figure 5). Results show that the model can accurately predict the actuator's bending angle and is thus useful for the selection of finger geometry and material during the design process. The higher bending rate of the small fingers indicates that they operate at lower pressures because their membrane is made of a softer material.

Using Equations (3) and (4), the inside surfaces of two facing fingers are then traced at various pressures to represent their workspace, adjust the initial angle (i.e., the relative position of the fingers as installed on the palm), and determine what size of objects can be gripped for a specific configuration (Figure 7). An object with a minimum diameter of approximately $40 \mathrm{~mm}$ can be enclosed by two facing fingers (120 mm long) with an initial angle of $35^{\circ}$ with the vertical. However, fingers only exert larger gripping forces when they interfere with the object that is grasped, i.e., for object diameters larger than $50 \mathrm{~mm}$. The maximum enclosed diameter is around $90 \mathrm{~mm}$ and is limited by the fingers' length. 


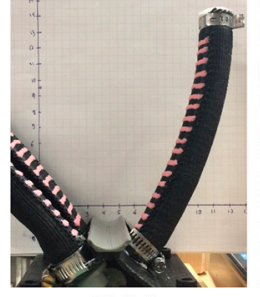

$69 \mathrm{kPa}$

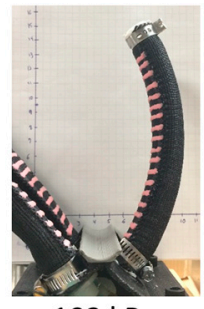

$103 \mathrm{kPa}$

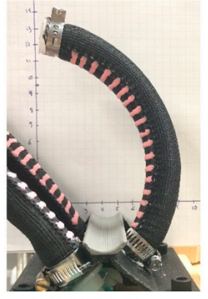

$138 \mathrm{kPa}$

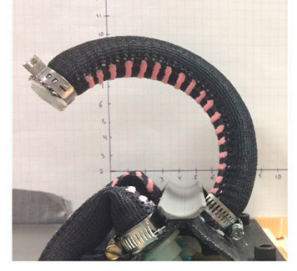

$172 \mathrm{kPa}$

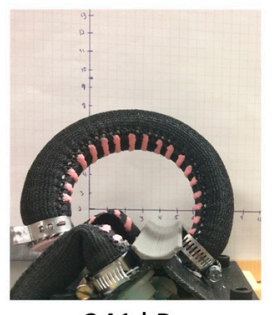

$241 \mathrm{kPa}$

Figure 6. Sleeved bending actuator under various operating pressures (small).

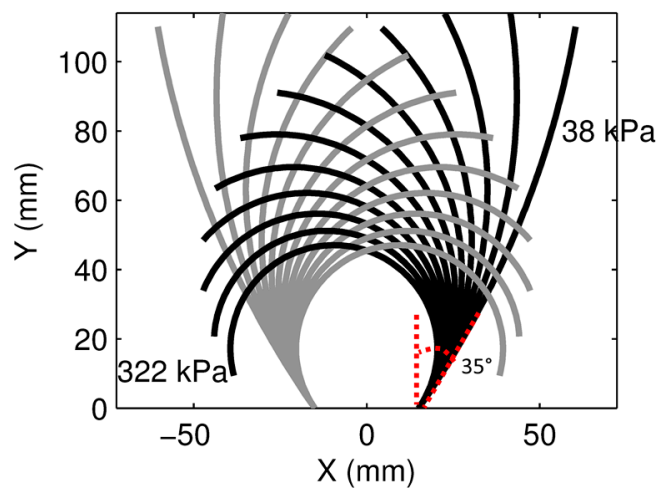

Figure 7. Two $120 \mathrm{~mm}$ facing fingers, traced for pressures ranging from $38 \mathrm{kPa}$ to $322 \mathrm{kPa}$. Only the inside surfaces of the fingers are traced for clarity (large gripper). The initial angle of the finger with the vertical is shown $\left(35^{\circ}\right)$.

\subsection{Payload Model}

A first order model based on energy conservation is derived to estimate the payload capacity of the proposed design (Figure 8). The bending actuator is considered to have a spring of stiffness of $k$, which deforms under a payload (force $F$ ). The model considers two states. In state 1 , the actuation pressure is $P_{1}$, and the actuator is in its free bending configuration (Figure $8 \mathrm{~b}$ ): Its radius $R_{1}$ and angle $\theta_{1}$ are determined using the model presented in Section 3.1. In state 2, the actuator is deformed by the payload (force $F$ ) until the force is applied directly at the tip of the finger. The payload is considered to be applied locally, vertically, and exactly in the center of the palm (at $x_{c}$ from the finger's base). In state 2, the actuator has a radius of $R_{2}$ and a bending angle of $\theta_{2}$. The pressure inside the finger is kept constant by a pressure regulator, leading to $P_{2}=P_{1}$. Considering each gripper's geometry, and especially the palm's width $\left(2 x_{c}\right)$, state 2 occurs when the actuators reach a radius $R_{2}$ of $81 \mathrm{~mm}$ and $65 \mathrm{~mm}$ for the small and large gripper, respectively.

Modelling the actuator as a linear spring of stiffness $k$, the work done by the payload force $F$ is defined as:

$$
\begin{gathered}
W_{i n}=-\int_{y=0}^{y=\Delta y} k y d y=-\frac{k \Delta y^{2}}{2}=-\frac{F \Delta y}{2} \\
\Delta y=Y_{2}-Y_{1}
\end{gathered}
$$


where $k$ is the apparent stiffness of the actuator in the direction of $F$, and $\Delta y$ is the vertical displacement of the actuator from state 1, where the "spring" is at equilibrium, to state $2 . \Delta y$ can be calculated from the position of the force application point at state $1\left(x_{c}, Y_{1}\right)$ and state $2\left(x_{c}, Y_{2}\right)$, using geometric relationships between $R_{1}$ or $R_{2}, x_{c}$, and the angle of the base $\left(55^{\circ}\right)$.

The output work (from the actuator's volume change) is defined as:

$$
W_{\text {out }}=\int_{V_{1}}^{V_{2}} P_{1} d V=P_{1}\left(V_{2}-V_{1}\right) \text { where } V_{2}<V_{1}
$$

Equating Equations (5) and (6) for energy conservation, the payload force $F$ can be determined for a given operating pressure. The model neglects the friction between the fingers and the object, and it assumes a linear stiffness and a single vertical contact point between the payload and the fingers. The payload experiments and results are described in the next section.

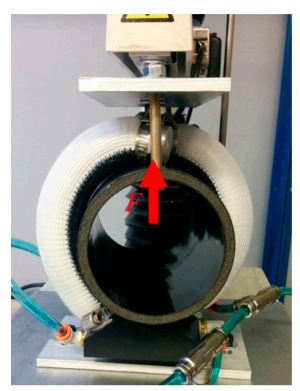

a

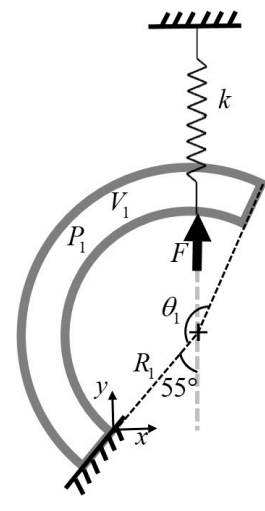

b (State 1)

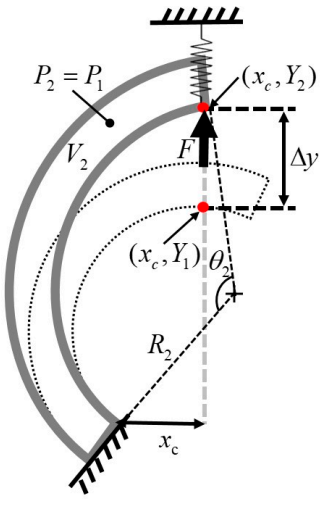

c (State 2)

Figure 8. Payload model. (a) Location of the payload force application point in the physical gripper; (b) State 1 and (c) State 2 of the actuator's payload model.

\section{Experiments and Results}

\subsection{Payload}

Maximum gripping (horizontal) and pull-out (vertical) payload capabilities of both grippers are characterized through two distinct experiments (Figure 9). For all experiments described in this paper (except drone experiments), pressure is controlled by a proportional regulator (Festo type VPPM).

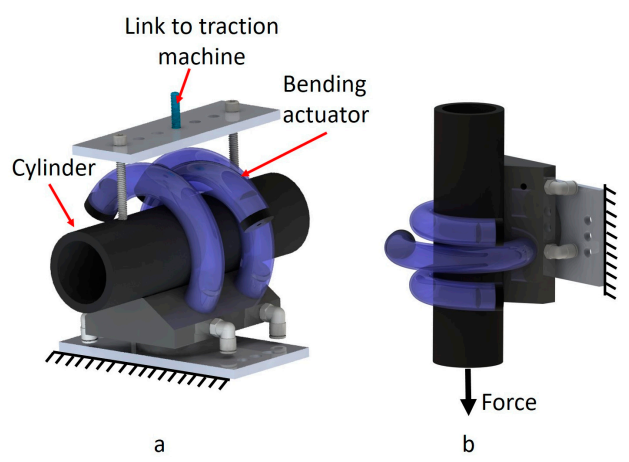

Figure 9. Experimental set-ups (a) to measure gripping payload, using a TAXT Plus Texture Analyzer (traction machine) (Stable Micro Systems, Godalming, Surrey, UK) with a $50 \mathrm{~kg}$ load cell and (b) to measure the pull-out force using free weights. Bending actuators are shown without their sleeves. 


\subsubsection{Gripping Payload}

In this test, the gripper encloses an ABS cylinder that is attached to the load cell of a TAXT Plus Texture Analyzer (Stable Micro Systems, Godalming, Surrey, UK) traction machine with a $50 \mathrm{~kg}$ load cell, and the load is perpendicular to the axis of the cylinder (Figure 9a). The maximum payload is considered to be the force at which a slope variation occurs in the force-to-displacement graph, i.e., when the cylinder slips through the fingers. Gripping payload depends on both the friction coefficient, which limits slippage between fingers and object, and the fingers' resistance to unwrapping (i.e., their stiffness).

Both grippers are tested with a $61 \mathrm{~mm}$ cylinder, a size that is fully enclosed by the fingers, and an $89 \mathrm{~mm}$ cylinder, in which case the fingertips barely touch. The small gripper can hold up to $52 \mathrm{~N}$ for a $61 \mathrm{~mm}$ cylinder (Figure 10), which is over the requirement for supporting the weight of the Phantom 2. However, it cannot support a relevant load when the diameter of the cylinder approaches its wrapping capacity $(89 \mathrm{~mm})$. The small gripper demonstrates a high payload-to-weight ratio of 46 , reinforcing its adequacy for flying machines.

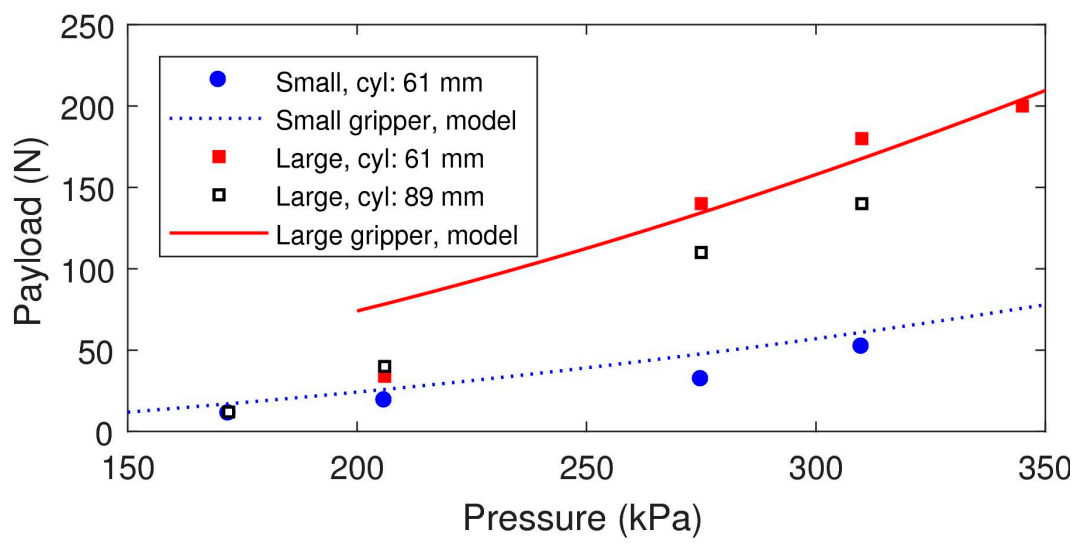

Figure 10. Gripping payloads (experimental and model predictions) for both grippers (small and large) at various operating pressures for two cylinder diameters. The lowest payload of three experiments for each set of conditions is shown.

The large version holds loads up to $200 \mathrm{~N}$ with a $61 \mathrm{~mm}$ cylinder, and, due to the increased stiffness of its fingers, it can also hold the larger cylinder $(89 \mathrm{~mm})$ with a $140 \mathrm{~N}$ load (Figure 10). The large gripper shows a lower payload-to-weight ratio (33.6) than the small gripper due to its heavy palm (not optimized for mass considerations). Both grippers still show high payload-to-weight ratios.

For the small fingers, experimental payloads are lower than model predictions (Figure 10), which can be caused by the assumptions of the model. For the large fingers, experiments show that the model can predict payloads with a mean error of $4.3 \%$ over $250 \mathrm{kPa}$. The simplified payload model is not exact, although it is a design tool that can be used to compare actuators made of different materials with various geometries and gripper configurations, as demonstrated for two distinct finger designs.

\subsubsection{Pull-Out}

Pull-out resistance measures the capacity of the gripper to retain an object under an axial force (Figure 9b). An ABS cylinder is placed in the gripper and loaded axially until it begins to slip, i.e., when the pull-out force is greater than the friction between the object and fingers. In the case of the small gripper, the small diameter and lower stiffness of the fingers cause some flexion at their base, limiting the force that can be applied to $20 \mathrm{~N}$ (Table 2). The increased wrapping angle and larger contact area between the fingers and the smaller ABS cylinder leads to a higher slip force.

For the large gripper, an increased cylinder diameter $(89 \mathrm{~mm})$ offers a greater slip-out force, up to $89 \mathrm{~N}$ instead of $64 \mathrm{~N}$ for the $61 \mathrm{~mm}$ cylinder (Table 2). The trend is only seen on the large gripper, 
because its bending radius does not make for a sufficient interference with the $61 \mathrm{~mm}$ cylinder, leading to a lower contact (friction) force.

Table 2. Slip test results for both grippers with two ABS cylinder diameters. Test pressure is indicated in brackets.

\begin{tabular}{ccc}
\hline ABS Cylinder Diameter & Small Fingers & Large Fingers \\
\hline \multirow{2}{*}{$61 \mathrm{~mm}$} & $20 \mathrm{~N}(275 \mathrm{kPa})$ & $28 \mathrm{~N}(275 \mathrm{kPa})$ \\
& & $64 \mathrm{~N}(310 \mathrm{kPa})$ \\
\hline \multirow{2}{*}{$89 \mathrm{~mm}$} & \multirow{2}{*}{$16 \mathrm{~N}(275 \mathrm{kPa})$} & $83 \mathrm{~N}(275 \mathrm{kPa})$ \\
& & $89 \mathrm{~N}(310 \mathrm{kPa})$ \\
\hline
\end{tabular}

\subsection{Durability}

Durability is experimentally validated by cyclically wrapping fingers around ABS cylinders. As durability experiments take weeks to complete, one test condition is chosen for each design. Cycling is controlled through Labview, using solenoid valves from Festo (type VUVG), a pressure regulator (Festo type VPPM), and an absolute pressure sensor (MediaSensor P51-50-A from SSI Technologies, Janesville, WI, USA), installed just before the gripper. Cycles are generated through a simulated square wave (on/off), sent to the solenoid valve, and counted only when validated by the pressure sensor's readings. Tests end when a finger suffers a failure (puncture), which prevents the pneumatic system from reaching the command pressure. Real-time video monitoring allows remote control, while time-stamped data, saved every $10 \mathrm{~min}$, allow recovery in case of power failure.

\subsubsection{Durability Experiments and Results}

Small bending actuators are tested by wrapping them around a $61 \mathrm{~mm}$ cylinder at $172 \mathrm{kPa}$. The frequency is kept low $(0.25 \mathrm{~Hz}$, i.e., one cycle per $4 \mathrm{~s})$ to avoid material heating due to viscoelastic effects, and the membrane has a maximum of 50\% elongation. After 700,000 cycles, the three bending actuators were still operational. Sleeves show signs of stretching, but membranes appear intact, aside from imprints of the seam and the elastic part of the sleeve in the membrane (Figure 11a).

Large bending actuators are tested under similar conditions, but also around an $89 \mathrm{~mm}$ cylinder at $206 \mathrm{kPa}$, to provide a 50\% maximum elongation on the outside of the bend. Two bending actuators reached 216,000 and 310,000 cycles, respectively, before their membrane suffered a puncture causing air leakage.

In both cases, the membrane puncture occurred on the outside of the bend, where the stretch is maximum (location is shown on Figure 4). The crack initiated on the surface and propagated through the membrane (Figure 11b) and appears to have been caused by surface abrasion. Observation under an optical microscope (Leica MZFLIII, Meyer Instruments Inc., Houston, TX, USA) reveals white fibers (from the elastic band) in multiple locations, slightly embedded in the membrane (Figure 11c). These fibers likely caused surface abrasion, which eventually cut through the membrane. The silicone used for the membrane has a durometer of 35 Shore A and is known to suffer almost instantaneous propagation once a visible crack is present [11]. Observation also shows imprints of the sleeve pattern and seam in the membrane.

\subsubsection{Durability Analysis}

Bending actuators are designed following principles proven to increase the life of extensible pneumatic artificial muscles [11]. It was thus expected that durability would be comparable between the extensible and large bending actuators, made of the same silicone, under similar tests conditions.

In the case of extensible muscles [11], failure after more than 200,000 cycles at $50 \%$ engineering strain was attributed to repeated contact with the sleeve's seam, causing surface defects. Large uniaxial 
strains contributed to the opening and propagation of newly formed surface defects until the puncture of the membrane occurred.

The location of the seam of the bending actuators, at the neutral axis, prevented a similar failure caused by large strains under the seam. In the case of bending actuators, it was the surface damage caused by the woven sleeve itself (Figure 11c) that led to the failure, although extensible actuators (from [11]) also showed an imprint of the sleeve pattern on the membrane. The fatigue life of the bending actuators is $15 \%$ higher than that of extensible actuators, leading to the conclusion that for this membrane material, fatigue failure is dictated by high strains, aggravating surface damages.

Small fingers have a lower durometer (25 Shore A), which translates into a lower modulus and, thus, operating pressure. Our hypothesis is that the combination of softer material and lower pressures minimizes the effect of local indentations caused by sleeve debris (fibers) or the seam. This could explain why, for identical strains, the small fingers are more than twice as durable as the bigger, stiffer actuators. The membrane's material is thus of the utmost significance, and an in-depth study of abrasion resistance of various elastomers would be relevant as future work.

Fatigue results show that, despite sleeved bending actuators resisting a large number of cycles due to their more uniform reinforcement, contact between the membrane and its reinforcement is still the cause of membrane failure. Their longer fatigue life, however, demonstrates that sleeved bending actuators can be both strong and durable.

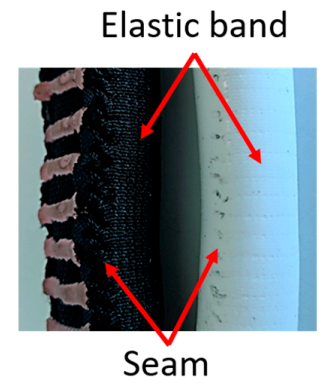

a

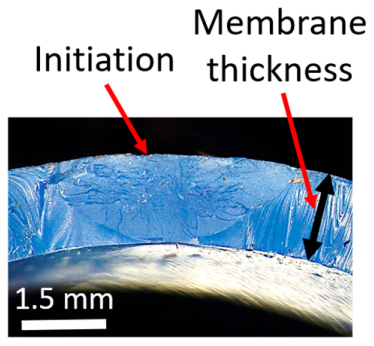

b

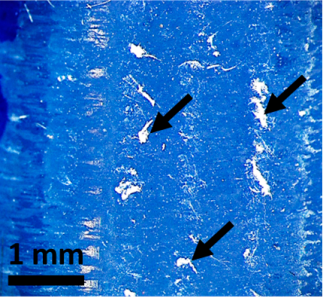

C

Figure 11. Visual (a) and optical microscope $(\mathbf{b}, \mathbf{c})$ analysis of actuator failure. (a) Sleeve and membrane of a small actuator, still operational after 700,000 cycles. Sleeve and seam imprints are visible in the membrane. (b) Section view of the crack in a large bending actuator. Initiation began on the outside surface of the membrane and propagated through the membrane, forming a semi-circular pattern (magnification: 20). (c) White fibers are embedded in the external surface of the large bending actuator. The picture was taken near the crack, i.e., in the middle of the actuator (magnification: 16).

\subsection{Applications}

\subsubsection{Drone Perching (Small Gripper)}

Integrating grippers into drones has been shown to have multiple applications, including surveillance missions, but also catching payloads in flight [30], perching for charging on lampposts and power lines [26,31], and payload carrying using arms [32] or electroadhesion [33]. Specific advantages of integrating a soft gripper into a drone are the resistance to damage, low weight, adaptability to the environment, and ability to avoid damaging fragile interactors.

The small gripper was integrated into a Phantom 2 quadcopter. The gripper is powered by a 16-g carbon dioxide cartridge equipped with a pressure regulator (16 g Micro Rock Regulator, Palmer Pursuit, North Highlands, CA, USA) to lower the pressure to around $310 \mathrm{kPa}$ (approx. 50 cycles/cartridge). The pneumatic circuit is designed using two miniature solenoid valves from Parker (X-Valve 912-000001-019) to provide a normally closed circuit that allows the gripper to remain closed without energy input. The total mass of the gripper (112.6 g), landing gear (92 g), pneumatics (142.5 g), and electronics ( $33.7 \mathrm{~g}$ ) is $380.8 \mathrm{~g}$, which is under the $400 \mathrm{~g}$ maximum. 
First, a static experiment validates that the drone can safely perch on a protruding $70 \mathrm{~mm}$ diameter cylinder, including perching at various angles (up to $45^{\circ}$ with the vertical) (Figure 12). This is critical to avoid flipping upside-down, in which case take-off would be impossible.

Then, the drone is flown inside a controlled environment and perched on a tree branch. The drone can perch, be powered off, stand still, then be powered back on, and take off from a tree branch (see Supplementary Material Video S1). The drone is also tested to be able to perch on the top of a fence, a square barrier, and a gas pipe (see Supplementary Material Video S1). These experiments show that the gripper offers multiple perching capabilities, adapting to various diameters, surfaces, and shapes, while having a simple and economical construction.

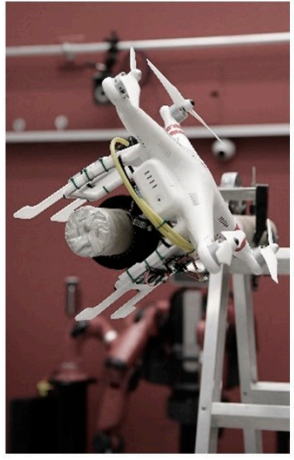

a

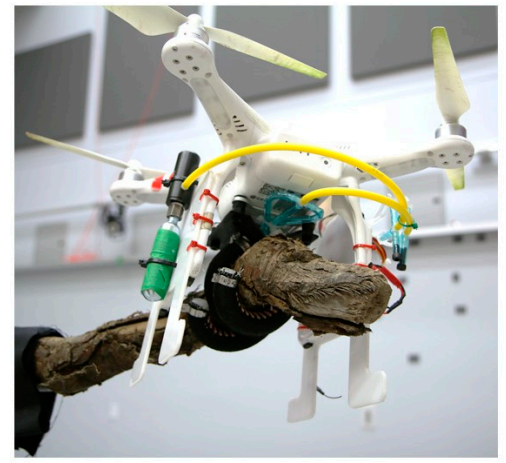

b

Figure 12. (a) Inclined perching at $45^{\circ}$ shows that the gripper can hold the drone steady on a uniform (low-friction) perch; (b) The drone can perch on a winding tree branch. See Video S1 (Supplementary Material).

\subsubsection{Food Manipulation (Small Gripper)}

Food manipulation is one of the best-known applications of soft grippers, as demonstrated by the company, Soft Robotics, for pick-and-place automation in the food industry [9]. The small gripper is tested by gripping food items of various shapes and textures using a constant operating pressure of $172 \mathrm{kPa}$ (Figure 13). The flexibility at the base of the fingers, a downfall for high payload slip tests, comes as an advantage to adapt to the uneven shapes of food items, such as broccoli or a bag of baby carrots (Figure 13). The gripper's adaptability makes holding these items possible without force sensors or specific programming.
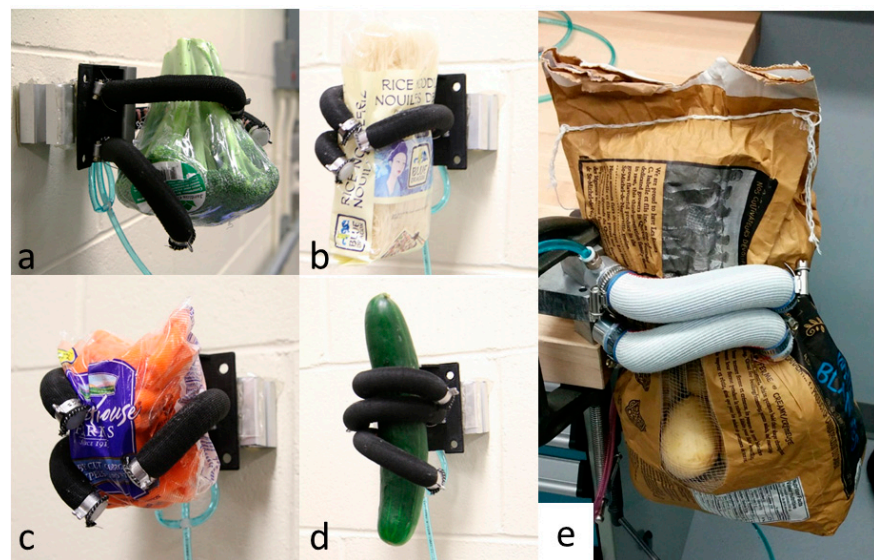

Figure 13. (a-d): Small gripper interacting with food items: (a) Broccoli; (b) rice noodles; (c) a bag of baby carrots; and (d) cucumber. The pressure is $172 \mathrm{kPa}$ for all tests. (e) The large gripper holding a 10-pound potato bag. 


\subsubsection{Heavy Load Manipulation (Large Gripper)}

The large gripper is appropriate for manipulating larger or heavier items of cylindrical or irregular shapes. During payload experiments (Section 4.1.1), the large gripper has shown that it can handle objects as large as $89 \mathrm{~mm}$ in diameter and loads of up to $200 \mathrm{~N}$ (Figure 10). A practical application of the large gripper is the gripping and holding of a 10-pound potato bag $(4.5 \mathrm{~kg})$, which has a highly irregular shape (Figure 13). The gripper can hold the bag despite the random placement of potatoes inside the bag.

\section{Conclusions}

Pneumatic soft grippers have lately been used to achieve clever new applications, such as collecting reef samples and assisting hand rehabilitation. Their widespread use is, however, limited by a compromise between payload capacity, requiring higher strains and pressure, and durability. This paper presented the design and testing of sleeved bending actuators for high force density gripping applications. The sleeved actuator integrates design principles that have been shown to increase the fatigue life of extensible muscles. The sleeve is the key to distributing pressure on a large and continuous area, which avoids local stress-raisers, leading to premature membrane puncture, as commonly seen in fiber-wrapped bending actuators. The sleeve also supports more pressure than the elastomer alone, thus increasing the payload, and protects the membrane from sharp objects.

It has been shown that gripper performances can be adapted to the desired application by changing the membrane's material, the fingers' geometry, and the palm configuration, using simplified models to predict the bending and payload in the design phase. A small and a large gripper show maximum payloads of up to $5.2 \mathrm{~kg}$ and $20 \mathrm{~kg}$, respectively. Bending actuators show a fatigue life of more than 700,000 cycles (small actuators) and 200,000 cycles (large actuators), which is a significant improvement on existing soft bending actuators, showing either durability or force, but not both. Application experiments demonstrate the versatility and adaptability of soft grippers, including drone perching, food item manipulation, and the handling of heavy, irregular packages, such as a 10-pound potato bag. In particular, drone perching is a promising avenue, allowing drones to be sent to remote sites in recognition or surveillance missions, without the limitation of short flight times.

Despite their force and durability performances, sleeved bending actuators still require further development to reach the technology readiness required for commercialization. In particular, durability could be further improved by choosing industrial-grade materials (elastic band, strain-limiting layer, and membrane) with automated sleeve sewing and assembly, as well as overmolding of the sleeve to reduce relative movements. In addition, specially designed fingertips could facilitate the pick-up of objects on the ground while maintaining power grasping capabilities. Regarding materials, an in-depth study of silicone elastomers resistance to abrasion would be relevant to select the most durable membrane materials.

Sleeved bending actuators are simple, low-cost, strong, versatile, and durable actuators for soft grippers, paving the way for the development of game-changing applications in soft robotics.

Supplementary Materials: The following are available online at https:/ / zenodo.org/ record/1313485\#.W02281KSCJ0: Video S1: Sleeved bending actuators for soft grippers-Applications.

Author Contributions: Conceptualization, G.M.; Investigation, G.M. and B.B.; Methodology, G.M.; Resources, B.B.; Supervision, J.-S.P.; Validation, G.M.; Writing—original draft, G.M.; Writing—review and editing, G.M. and J.-S.P.

Acknowledgments: The authors would like to thank John Bass for piloting the Phantom 2. Geneviève Miron received personal funding in the form of a scholarship from NSERC (National Science and Engineering Research Council). Most pneumatic control equipment was graciously sponsored by Festo.

Conflicts of Interest: The authors declare no conflict of interest. 


\section{References}

1. Amend, J.R.; Brown, E.; Rodenberg, N.; Jaeger, H.M.; Lipson, H. A positive pressure universal gripper based on the jamming of granular material. IEEE Trans. Robot. 2012, 28, 341-350. [CrossRef]

2. Manti, M.; Hassan, T.; Passetti, G.; D'Elia, N.; Laschi, C.; Cianchetti, M. A Bioinspired Soft Robotic Gripper for Adaptable and Effective Grasping. Soft Robot. 2015, 2, 107-116. [CrossRef]

3. Polygerinos, P.; Lyne, S.; Wang, Z.; Nicolini, L.F.; Mosadegh, B.; Whitesides, G.M.; Walsh, C.J. Towards a soft pneumatic glove for hand rehabilitation. In Proceedings of the IEEE International Conference on Intelligent Robots and Systems, Tokyo, Japan, 3-7 November 2013; pp. 1512-1517.

4. Shintake, J.; Rosset, S.; Schubert, B.; Floreano, D.; Shea, H. Versatile Soft Grippers with Intrinsic Electroadhesion Based on Multifunctional Polymer Actuators. Adv. Mater. 2016, 28, 231-238. [CrossRef] [PubMed]

5. Hubbard, A.M.; Mailen, R.W.; Zikry, M.A.; Dickey, M.D.; Genzer, J. Controllable curvature from planar polymer sheets in response to light. Soft Matter 2017, 13, 2299-2308. [CrossRef] [PubMed]

6. Hensel, R.; Moh, K.; Arzt, E. Engineering Micropatterned Dry Adhesives: From Contact Theory to Handling Applications. Adv. Funct. Mater. 2018, 28, 1800865. [CrossRef]

7. Galloway, K.C.; Polygerinos, P.; Walsh, C.J.; Wood, R.J. Mechanically programmable bend radius for fiber-reinforced soft actuators. In Proceedings of the 16th International Conference on Advanced Robotics (ICAR 2013), Montevideo, Uruguay, 25-29 November 2013; pp. 1-6.

8. Shintake, J.; Cacucciolo, V.; Floreano, D.; Shea, H. Soft Robotic Grippers. Adv. Mater. 2018, 30, 1707035. [CrossRef] [PubMed]

9. Soft Robotics. Technology—Soft Robotics. 2017. Available online: https://www.softroboticsinc.com/technology/ (accessed on 10 April 2017).

10. Galloway, K.C.; Becker, K.P.; Phillips, B.; Kirby, J.; Licht, S.; Tchernov, D.; Wood, R.J.; Gruber, D.F. Soft Robotic Grippers for Biological Sampling on Deep Reefs. Soft Robot. 2016, 3, 23-33. [CrossRef] [PubMed]

11. Miron, G.; Plante, J. Design Principles for Improved Fatigue Life of High-Strain Pneumatic Artificial Muscles. Soft Robot. 2016, 3, 177-185. [CrossRef]

12. Voica, A. Solving the Challenges of Robotic Picking-Ocado Technology. Ocado Technology. 2016. Available online: http:/ / ocadotechnology.com/blog/solving-the-challenges-of-robotic-picking/ (accessed on 11 August 2017).

13. Taylor Farms. Taylor Farms Innovates with Soft Robotics. 2016. Available online: http:/ /www.taylorfarms. com/news / taylor-farms-innovates-soft-robotics / (accessed on 11 August 2017).

14. Yap, H.K.; Ng, H.Y.; Yeow, C.-H. High-Force Soft Printable Pneumatics for Soft Robotic Applications. Soft Robot. 2016, 3, 144-158. [CrossRef]

15. Patel, D.K.; Sakhaei, A.H.; Layani, M.; Zhang, B.; Ge, Q.; Magdassi, S. Highly Stretchable and UV Curable Elastomers for Digital Light Processing Based 3D Printing. Adv. Mater. 2017, 29, 1606000. [CrossRef] [PubMed]

16. Guo, J.; Elgeneidy, K.; Xiang, C.; Lohse, N.; Justham, L.; Rossiter, J. Soft pneumatic grippers embedded with stretchable electroadhesion. Smart Mater. Struct. 2018, 27, 55006. [CrossRef]

17. Martinez, R.V.; Glavan, A.C.; Keplinger, C.; Oyetibo, A.I.; Whitesides, G.M. Soft actuators and robots that are resistant to mechanical damage. Adv. Funct. Mater. 2014, 24, 3003-3010. [CrossRef]

18. Mosadegh, B.; Polygerinos, P.; Keplinger, C.; Wennstedt, S.; Shepherd, R.F.; Gupta, U.; Shim, J.; Bertoldi, K.; Walsh, C.J.; Whitesides, G.M. Pneumatic Networks for Soft Robotics that Actuate Rapidly. Adv. Funct. Mater. 2014, 24, 2163-2170. [CrossRef]

19. Polygerinos, P.; Wang, Z.; Overvelde, J.T.; Galloway, K.C.; Wood, R.J.; Bertoldi, K.; Walsh, C.J. Modeling of Soft Fiber-Reinforced Bending Actuators. IEEE Trans. Robot. 2015, 31, 778-789. [CrossRef]

20. Natividad, R.; del Rosario, M.; Chen, P.C.Y.; Yeow, C.-H. A Reconfigurable Pneumatic Bending Actuator with Replaceable Inflation Modules. Soft Robot. 2018, 5, 304-317. [CrossRef] [PubMed]

21. Al-Fahaam, H.; Davis, S.; Nefti-Meziani, S. The design and mathematical modelling of novel extensor bending pneumatic artificial muscles (EBPAMs) for soft exoskeletons. Robot. Autom. Syst. 2018, 99, 63-74. [CrossRef] 
22. Klute, G.K.; Hannaford, B. Fatigue characteristics of McKibben artificial muscle actuators. In Proceedings of the 1998 IEEE/RSJ International Conference on Intelligent Robots and Systems-Innovations in Theory, Practice and Applications, Victoria, BC, Canada, 17 October 1998; Volume 3, pp. 1776-1781.

23. Kingsley, D.A.; Quinn, R.D. Fatigue life and frequency response of braided pneumatic actuators. In Proceedings of the 2002 IEEE International Conference on Robotics and Automation, Washington, DC, USA, 11-15 May 2002; Volume 3, pp. 2830-2835.

24. Nithi-Uthai, N.; Laksanacharoen, S. Extending fatigue life of McKibben artificial muscle actuators. In Proceedings of the Minerals, Metals and Materials Society-The 3rd International Conference on Processing Materials for Properties, Bangkok, Thailand, 7-10 December 2008; Volume 1, pp. 453-456.

25. FESTO. Fluidic Muscle DMSP/MAS. 2016. Available online: https://www.festo.com/cat/en-us_us/data/ doc_enus/PDF/US/DMSP-MAS_ENUS.PDF (accessed on 29 August 2017).

26. Hambling, D. Swarm Troopers: How Small Drones will Conquer the World, 1st ed.; Archangel Ink: Venice, FL, USA, 2015.

27. Cutkosky, M.R.; Howe, R.D. Human Grasp Choice and Robotic Grasp Analysis. In Dextrous Robot Hands; Springer: New York, NY, USA, 1990; pp. 5-31.

28. National Aeronautics and Space Administration. Human Performance Capabilities; NASA: Washington, DC, USA, 1995.

29. Gent, A.N. On the Relation between Indentation Hardness and Young's Modulus. Rubber Chem. Technol. 1958, 31, 896-906. [CrossRef]

30. Thomas, J.; Polin, J.; Sreenath, K.; Kumar, V. Avian-Inspired Grasping for Quadrotor Micro UAVs. In Proceedings of the ASME 2013 International Design Engineering Technical Conferences and Computers and Information in Engineering Conference, Portland, OR, USA, 4-7 August 2013; p. V06AT07A014.

31. Gentry, N.; Hsieh, R.; Nguyen, L. Multi-use UAV Docking Station Systems and Methods. U.S. Patent US9387928 B1, 18 December 2016.

32. Prodrone. PRODRONE Unveils the World's First Dual Robot Arm Large-Format Drone. 2016. Available online: https:/ / www.prodrone.jp/en/archives/1420/ (accessed on 5 May 2017).

33. Grabit Inc. Drone Delivery with Grabit Electroadhesion Grippers-YouTube. 2014. Available online: https: / / www.youtube.com/watch?v=w4COP3TJsrk (accessed on 5 May 2017).

(C) 2018 by the authors. Licensee MDPI, Basel, Switzerland. This article is an open access article distributed under the terms and conditions of the Creative Commons Attribution (CC BY) license (http:/ / creativecommons.org/licenses/by/4.0/). 